\title{
Cooperation Between a Doctor and a Podiatrist to Improve the Quality of Life of Patients with Ingrown Toenails
}

\author{
Tomasz Trochanowski ${ }^{1}$, Ewa Baum ${ }^{2},{\text { Ryszard } \dot{Z} a b a^{3}}^{3}$ \\ 1 Zrodlana Medical Centre, Zielona Gora, Poland \\ 2 Department of Social Sciences and the Humanities, Poznan University of Medical Sci- \\ ences, Poland \\ 3 Department of Dermatology and Venereology, Poznan University of Medical Sciences, \\ Poland
}

\begin{abstract}
Cooperation between doctors of various specialties and other medical specialists is the standard of care in the treatment of patients. Due to the variety of diseases and the dynamic development of medicine in general, it is difficult to be an expert in every field and know all the recommended treatments. An example of such cooperation is the joint treatment of patients with the problem of ingrown toenails. The article contains an analysis of patients who received treatment in a doctor's office in cooperation with podiatrists. A conservative approach towards the treatment of this condition sees the patient being initially diagnosed and treated by a podiatrist and then later, if necessary, being referred to a doctor for surgical treatment. The exchange of experiences and information on patients treated by interdisciplinary teams allows doctors and podiatrists to find the best possible treatment and improve the quality of life of patients. The follow-up of patients after surgery can be performed later in podiatry offices. Owing to modern electronic communication, it is possible for a doctor to constantly monitor the patient's condition without the need for direct visits to the doctor's office.
\end{abstract}

\section{Introduction}

It is a commonly accepted standard among physicians to refer patients to other specialist physicians in the case of a diagnosis or a suspicion of a disease that requires expert treatment. This procedure makes it possible to treat patients in accordance with the latest available standards. An example may be the treatment of diseases of the nervous system, which although usually performed by neurologists, is managed in cooperation with specialists such as psychiatrists and internists. Additionally, medical professionals other than doctors can bring indisputable benefits as far as therapy 


\section{Tomasz Trochanowski, Ewa Baum, Ryszard Żaba}

outcomes are concerned and generally supplement the method of treatment with new solutions (Gilhus et al., 2011). Medical professionals other than doctors are all those medical specialists that contribute to the overall process of treatment, i.e. nurses, psychologists, speech therapists, occupational therapists, and physical therapists (Busari et al., 2017; Chomienne et al., 2011; Fagin, 1992; Kone et al., 2018; Stratil et al., 2017). They can all work together in teams, e.g. in hospital rehabilitation units, where the success of the rehabilitated patient depends on close cooperation between all the team members (Kiwerski, 2021). However, such cooperation also functions outside the hospital environment, as many patients are treated on an outpatient basis. An example is the cooperation of doctors and podiatrists in the treatment of podological diseases (Bandyk, 2018; Menz et al., 2020; Turner et al., 2020).

Podiatrists, i.e. foot disease specialists, appeared in Poland as a completely new medical profession relatively recently, compared to countries such as Germany, Great Britain or Spain, where the system of vocational and academic training for podiatrists had existed for many years. However, both in the countries with an established professional legacy of podiatrists and in Poland, a podiatrist is not always the specialist to whom patients first turn in the event of a foot disorder (Ratajczak et al., 2021). Usually, patients choose a GP or the relevant specialist, despite the fact that it is the podiatrist who diagnoses a disease and then treats it or refers the patient to another medical specialist. On the other hand, although a doctor visited by a patient with a foot condition may treat them in their office, they also have the option of referring the patient to a podiatrist, despite having the appropriate competences to perform the treatment. Doctors can do this for several reasons such as insufficient time to undertake the therapy in their office, a lack of specialist podiatry equipment that makes it impossible to start first-line treatment in the doctor's office and, finally, the trust in the competences and knowledge of podiatrists.

Currently, there are many instances of cooperation between podiatrists and physicians as well as medical specialists in other fields (Bandyk, 2018; Menz et al., 2020; Turner et al., 2020). Foot defects are diagnosed by doctors and physiotherapists, while podiatrists remove calluses and corns before selecting the appropriate insoles. Diabetic foot can be treated jointly by doctors (diabetologists, surgeons) and podiatrists; similarly, onychomycosis can be treated by a dermatologist and a podiatrist. In such cases, doctors deal with the appropriate pharmacotherapy and the treatment of acute disorders, while the podiatrist is responsible for regular follow-ups and the proper education of patients in the field of foot care. 
Cooperation between a doctor and a podiatrist may also concern a common problem such as an ingrown toenail (Haneke, 2012; Heidelbaugh \& Lee, 2009; Mayeaux et al., 2019; Trochanowski et al., 2019). When dealing with this disease, doctors usually use surgical methods from the very beginning. It is not necessarily the best solution for a patient whose stage of disease enables the use first-line treatment, which is associated with fewer resulting complications. A podiatrist should be able to treat an uncomplicated case of an ingrown nail, starting the therapy with the most basic methods of podiatric treatment, such as inserting a tamponade under the edge of the nail, setting an appropriate nail brace, using tapes to lower the nail folds, or performing a reconstruction of the nail plate using Arkada's method (Haneke, 2012; Trochanowski et al., 2019). If the above-mentioned podiatric treatment is not successful, the podiatrist should refer the patient to a doctor in order to consider surgical treatment.

\section{Materials and Methods}

The analysis performed in this study covers a period of one year, from November 2020 to October 2021, and concerns 157 new patients admitted to doctor's offices for consultation or treatment of ailments related to ingrown toenails. The relationships were tested with Chi-squared tests with Yates's correction and Chi-squared tests at the significance level of $\alpha=0.05$, using Dell Inc. software (2016), i.e. Dell Statistica (a data analysis software system), version 13, software.dell.com. The authors reviewed the scientific literature according to the PRISMA protocol. PubMed, Google Scholar, and ResearchGate databases were searched using medical search terms, i.e. medical cooperation, ingrown toenail, podiatry, telemedicine, and quality of life. Ultimately, over a dozen scientific articles were selected, including review papers, case stages, and retrospective studies. The authors' own experiences related to various aspects of cooperation with podiatrists and the treatment and contact with patients complaining of ingrown toenails were also taken into account.

\section{Results}

Of the total number of 157 new patients in doctor's offices, 134 made an appointment by referral from a podiatrist, while 23 did so directly, without prior instruction. In the first group of patients (after referral), 


\section{Tomasz Trochanowski, Ewa Baum, Ryszard Żaba}

$56 \%$ were treated with plastic surgery (correction) of the nail folds combined with reconstruction of the plate using Arkada's method; in the second group (without referral), this procedure was performed in $26 \%$ of patients $(p=0.008)$. A number of patients $(20 \%)$ from the group referred to a doctor by podiatrists for further treatment had previously undergone treatment with Arkada's method. Thus, only the surgical part of the comprehensive plastic surgery of nail folds was performed in the doctor's office. In total, plastic surgery was performed in $76 \%$ of patients from the group referred by podiatrists.

In the group of referred patients, $21 \%$ underwent only podiatric procedures (basic treatment, Arkada's method), compared to $52 \%$ in the group of patients whose appointment took place without a previous referral from the podiatrist $(p=0.003)$. After plastic surgery of nail folds was performed in 102 patients from the group referred by podiatrists, 75 of them returned to their original podiatric offices. The percentage of patients who underwent further treatment at podiatrists' offices was higher in the first group (referred patients) compared to those who made their appointments directly: $56 \%$ and $17 \%$, respectively $(p=0.006)$.

Patients referred by podiatrists contacted their physicians before and after the procedures performed in the doctor's office $(96 \%$ and $93 \%$, respectively) much more often $(p<0.001)$ than the second group of patients (48\% and $52 \%)$.

\section{Discussion}

The topic of cooperation between doctors and other medical specialists appears in many studies; however, there is an apparent deficit of information about the cooperation of podiatrists and doctors. There are several reasons for this. Firstly, in many countries where the position of a podiatrist is established and the podiatric education system has been functioning for a long time, the podiatrist often has much broader competences to perform more specialized procedures, including those that require the use of local anesthesia. An example of such a country is the United Kingdom, where podiatrists often treat ingrown nails by excising the fragment or the entire nail and then use phenol to destroy the matrix. In addition, British podiatrists may additionally specialize in foot surgery, which makes it possible for them to perform procedures that are usually conducted by doctors. Furthermore, plastic surgery of nail folds in Poland and other countries is performed less frequently than the removal of the fragment or of the entire 
nail (Haneke, 2012; Heidelbaugh \& Lee, 2009; Trochanowski et al., 2019). The latter type of treatment is generally more easily accessible, is less timeconsuming to perform, and at the same time less expensive; however, this does not mean that it is more effective or aesthetically more satisfactory for the patient.

The lack of effectiveness and the unsatisfactory aesthetics of the toe and nail after removal procedures in surgical rooms is one of the reasons why podiatrists recommend surgical treatment performed by a specialist. The recommended specialists can perform another type of treatment, i.e. plastic surgery of nail folds. This treatment should result in permanent healing of the ingrown nail and a good aesthetic effect, thus ultimately improving the patient's quality of life. Such a situation, in which a podiatrist recommends a doctor who can perform specialized and targeted treatment, is beneficial both for the patient and the podiatrist themselves. First of all, having exhausted all the options of conservative treatment within their competence, the podiatrist should be aware of their limitations and refer patients to other specialists, as this reduces the risk of being accused of not exploiting the potential of other treatments. In this way, the patient is given the option of more effective therapies. Secondly, the patient's confidence in the podiatrist increases, despite the ineffectiveness of basic treatment in the podiatry office.

The results show the appropriateness of patient recommendations made by podiatrists, because the vast majority of patients in this group underwent treatment that could not be performed in a podiatry office. A smaller percentage of patients, previously treated in podiatric offices, underwent only further podiatric procedures in a doctor's office. However, these cases were mostly connected with the need for local anesthesia, which typically cannot be administered by a podiatrist.

The high percentage of patients who returned to podiatric offices after a specialist medical treatment could be explained by the fact that patients accept recommendations and understand the essence of cooperation between a podiatrist and a doctor. After the medical procedure is performed by a doctor, most patients return to podiatry in order to attend regular follow-ups and make a full recovery. This pattern of behavior ensures that the podiatry community is not wary of losing its patients despite referrals to other specialists, which, in the era of commercialization of services and a strong competition for patients, is also an important factor. Moreover, such post-operative care is often an optimal solution for the doctor; on the one hand, it ensures professional patient care provided by specialists until the patient is completely cured, on the other 


\section{Tomasz Trochanowski, Ewa Baum, Ryszard Żaba}

hand, it allows them to focus on other professional duties. However, the lack of patient follow-up after nail fold surgery in a doctor's office does not mean that there is no contact between the doctor and the patient. Even before the procedure, the doctor communicates with the patient referred to them by the podiatrist, using modern means of communication (email, SMS, social messaging). The patient sends photos of their sick nails to the doctor, together with basic information about the previously received treatment and the presence of current symptoms. The purpose of such communication is to confirm the correctness of the patient's qualification for surgical treatment and, possibly, to prevent disqualification upon arrival at the doctor's office, which is particularly important in the case of patients who come from distant regions. Again, it can be stated that the high percentage of medical procedures performed on the referred patients confirms the correctness of the described procedure. After the procedure is completed, communication between the patient and the doctor makes it possible to perform professional assessment of tissue healing and provides podiatrists with possible suggestions for further patient care, thus reducing the need for possible follow-ups at the doctor's office. Such a control system also makes it possible to treat patients from distant regions of the country and even from abroad, and the patients themselves are sure that their recovery is controlled both by the doctor who performed the procedure and the podiatrist to whom the patients eventually return.

The presented model of treatment, which takes place both in podiatric and in medical offices, proves effective for the patient due to the opportunity of receiving the best possible treatment. Moreover, when choosing a doctor's office, no patient has to limit themselves to the offices located close to their place of residence. The effectiveness of treatment and the good aesthetic effect of the cured toenail certainly improves the quality of life of the patient (Bengoa Vallejo et al., 2019). Such a model of cooperation is also possible owing to modern communication technologies, with communication taking place not only between the patient and the doctor, but also between the podiatrist and the doctor. Communication via email, SMS, or social messaging has existed for many years, but due to the COVID pandemic, it has become not only more widespread, but also more readily accepted by patients themselves (Kavitha et al., 2020; Neville et al., 2021). Traditional direct admission of patients is still the most common practice; however, the development of modern communication technologies and telemedicine will certainly continue (Corcoran et al., 2003). 
Cooperation Between a Doctor and a Podiatrist to Improve the Quality...

\section{Conclusions}

In order to treat a disease effectively, all the available methods of firstline treatment should be used. If there is no effect, specialist treatment should be applied. Therefore, it is important for doctors of various specialties and other medical specialists to cooperate, including podiatrists. This cooperation will almost certainly result in a better quality of treatment and hence increase the quality of life of patients. Moreover, modern means of electronic communication between specialists, as well as with patients, can serve to further improve the therapeutic process.

\section{R E F E R E N C E S}

Bandyk, D. F. (2018). The diabetic foot: Pathophysiology, evaluation, and treatment. Seminars in vascular surgery, 31(2-4), 43-48. https://pubmed.ncbi. nlm.nih.gov/30876640

Bengoa Vallejo, R. B., López López, D., Palomo López, P., Soriano Medrano, A., Morales Ponce, Á., \& Losa Iglesias, M. E. (2019). Quality of life improvement in aged patients after toenail surgery. Deutsche Gesellschaft für Gerontologie und Geriatrie, 52(8), 789-794. https://pubmed.ncbi.nlm.nih.gov/30710168

Busari, J. O., Moll, F. M., \& Duits, A. J. (2017). Understanding the impact of interprofessional collaboration on the quality of care: a case report from a smallscale resource limited health care environment. Journal of multidisciplinary healthcare, 10, 227-234. https://pubmed.ncbi.nlm.nih.gov/28652761

Chomienne, M. H., Grenier, J., Gaboury, I., Hogg, W., Ritchie, P., \& FarmanovaHaynes, E. (2011). Family doctors and psychologists working together: doctors' and patients' perspectives. Journal of evaluation in clinical practice, 17(2), 282-287. https://pubmed.ncbi.nlm.nih.gov/20874836

Corcoran, H., Hui, E., \& Woo, J. (2003). The acceptability of telemedicine for podiatric intervention in a residential home for the elderly. Journal of telemedicine and telecare, 9(3), 146-149. https://pubmed.ncbi.nlm.nih.gov/ 12877776

Fagin, C. M. (1992). Collaboration between nurses and physicians: no longer a choice. Academic medicine: journal of the Association of American Medical Colleges, 67(5), 295-303. https://pubmed.ncbi.nlm.nih.gov/1575859

Gilhus, N. E., Stovner, L .J., Gjerstad, L., \& Hoff, J. M. (2011). Partnership and cooperation is necessary to improve and expand neurology. Acta neurologica Scandinavica. Supplementum, 191, 1-4. https://pubmed.ncbi.nlm.nih.gov/21 711249

Haneke, E. (2012). Controversies in the Treatment of Ingrown Nails. Dermatology research and practice, 2012, 783924. https://pubmed.ncbi.nlm.nih.gov/2267 5345 


\section{Tomasz Trochanowski, Ewa Baum, Ryszard Żaba}

Heidelbaugh, J. J., \& Lee, H. (2009). Management of the ingrown toenail. American family physician, 79(4), 303-308. https://pubmed.ncbi.nlm.nih.gov/19235497

Kavitha, K. V., Deshpande, S. R., \& Pandit, A. P. (2020). Unnikrishnan AG. Application of tele-podiatry in diabetic foot management: A series of illustrative cases. Diabetes \& metabolic syndrome, 14(6), 1991-1995. https://pubmed. ncbi.nlm.nih.gov/33080541

Kiwerski, J. E. (2021). Scientific Idea of Professor Marian Weiss. Ortopedia, traumatologia, rehabilitacja, 23(3), 213-219. https://pubmed.ncbi.nlm.nih.gov/341 87941

Kone, I., Klein, G., Siebenhofer, A., Dahlhaus, A., \& Guethlin, C. (2018). GPs' assessment of cooperation with other health care providers involved in cancer care-a cross-sectional study. European journal of cancer care, 27(1). https://pubmed.ncbi.nlm.nih.gov/28983996

Mayeaux, E. J., Carter, C., \& Murphy, T. E. (2019). Ingrown Toenail Management. American family physician, 100(3), 158-164. https://pubmed.ncbi.nlm.nih. gov $/ 31361106$

Menz, H. B., Harrison, C., Britt, H., Whittaker, G. A., Landorf, K. B., \& Munteanu, S. E. (2020). Management of Hallux Valgus in General Practice in Australia. Arthritis care \& research, 72(11), 1536-1542. https://pubmed.ncbi. nlm.nih.gov/31549773

Neville, K., Black, A. T., \& Fridman, R. (2021). Epidemiological Survey of the Impact of COVID-19 on Telemedicine in the Practice of Foot and Ankle Surgery in the United States. The Journal of foot and ankle surgery, 60(3), 455-460. https://pubmed.ncbi.nlm.nih.gov/33518507

Ratajczak, P., Mutka, K., Michalak, M., Kopciuch,D., Paczkowska, A., Zaprutko, T., \& Kus, K. (2021). Patients' awareness of the prevention and treatment of the selected podiatry diseases. Journal of cosmetic dermatology. https://pubmed.ncbi.nlm.nih.gov/34591357

Stratil, J., Rieger, M. A., \& Voelter-Mahlknecht, S. (2017). Optimizing cooperation between general practitioners, occupational health and rehabilitation physicians in Germany: a qualitative study. International archives of occupational and environmental health, 90(8), 809-821. https://pubmed.ncbi.nlm.nih.gov/ 28681175

Trochanowski, T., Kotlega, D., \& Gołąb-Janowska, M. (2019). Plastic surgery combined with Arkada's method. Pomeranian Journal of Life Sciences, 65(2), 42-44. https://sciendo.com/pdf/10.21164/pomjlifesci.574

Turner, C., Gardiner, M. D., Midgley, A., \& Stefanis, A. (2020). A guide to the management of paediatric pes planus. Australian journal of general practice, 49(5), 245-249. https://pubmed.ncbi.nlm.nih.gov/32416653 